\title{
New window of opportunity with ICls in melanoma
}

Patients with resectable, stage III melanoma have a high risk of relapse after surgery. Thus, efforts have been made to improve outcomes using adjuvant therapies, historically immunotherapy with IFNa and more recently with immune-checkpoint inhibitors (ICIs), or targeted therapy for $B R A F^{\mathrm{V} 600 \mathrm{E} / \mathrm{K}}$-mutant disease.

Now, three studies reveal that the presurgical setting provides a window of opportunity to improve patient outcomes with ICIs.

A strong rationale favours the use of ICIs in the neoadjuvant rather than the adjuvant setting: a more productive antitumour immune response might ensue when the tumour and associated tumourinfiltrating lymphocytes (TILs) are present. In the OpACIN study, these two approaches were compared directly in 20 patients with stage IIIB-IIIC melanoma. Those in the adjuvant group received four courses of ipilimumab (ipi) at $3 \mathrm{mg} / \mathrm{kg}$ plus nivolumab (nivo) at $1 \mathrm{mg} / \mathrm{kg}$ after surgery. Patients in the neoadjuvant group received the same regimen but with two courses given before and two after surgery. Importantly, neoadjuvant therapy was feasible, with all patients undergoing surgery at the planned time point. Moreover, the pathological response rate (pRR) was $78 \%$, with pathological complete responses (pCRs) in 33\% of patients.

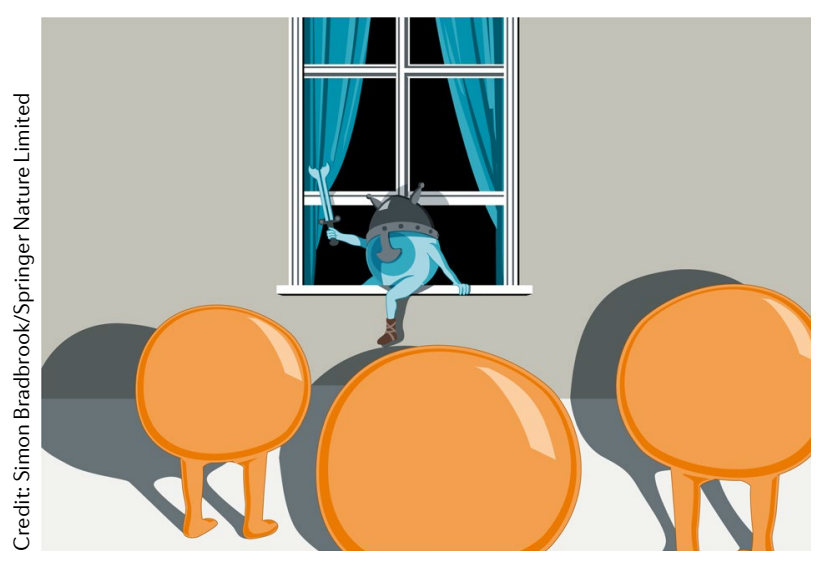

At a median follow-up duration of $\sim 26$ months, no patient with any pathological response had relapsed. By contrast, two patients without a pathological response had relapsed. These findings support a pathological response as a surrogate of favourable relapse-free survival (RFS). Overall, RFS at this time point was $80 \%$ in the neoadjuvant arm versus $60 \%$ in the adjuvant arm.

"Neoadjuvant immunotherapy was also superior to adjuvant immunotherapy in increasing the abundance of tumour-resident T cell clones in peripheral blood," states lead author Christian Blank. Accordingly, a greater number of newly detected circulating $\mathrm{T}$ cell clones after treatment, as well as baseline factors including a higher number of TILs, a less diverse TIL repertoire (perhaps indicating productive $\mathrm{T}$ cell clonality), and high expression of an IFN $\gamma$ gene signature, were identified as potential biomarkers of favourable RFS.

Many of these findings were recapitulated in a phase II study by Jennifer Wargo and colleagues. In this study, 23 patients with stage III melanoma received neoadjuvant treatment with up to three doses of ipi $(3 \mathrm{mg} / \mathrm{kg})$ plus nivo $(1 \mathrm{mg} / \mathrm{kg})$ or up to four doses of nivo $(3 \mathrm{mg} / \mathrm{kg})$ alone; the radiographic response rates were $73 \%$ versus $25 \%$, with pCR rates of $45 \%$ versus $25 \%$. Achieving a pCR was associated with favourable survival outcomes. Correspondingly, all survival outcomes were numerically superior with ipi plus nivo; all patients who received this regimen were alive at a median follow-up duration of $\sim 16$ months. The potential predictive biomarkers reported were similar to those identified in OpACIN.

"These trials demonstrate for the first time that ICI treatment before surgery is effective; however, regimens and biomarkers of a response need to be optimized, as not all patients benefit and the rate of adverse events (AEs) is high," Wargo summarizes. Indeed, across these two studies, the rates of grade $\geq 3$ AEs with ipi plus nivo were 73-90\% (versus only $8 \%$ with nivo alone).

"We have already conducted a subsequent trial of neoadjuvant ipi plus nivo, with the aim of reducing the toxicity but preserving the efficacy of treatment (OpACIN-neo), and I presented the preliminary data at the ESMO 2018 Congress," says Blank. In OpACIN-neo, 86 patients were treated with 2 doses of either ipi $(3 \mathrm{mg} / \mathrm{kg})$ plus nivo $(1 \mathrm{mg} / \mathrm{kg})$, ipi ( $1 \mathrm{mg} / \mathrm{kg})$ plus nivo $(3 \mathrm{mg} / \mathrm{kg})$, or ipi $(3 \mathrm{mg} / \mathrm{kg})$ alone directly followed by 2 doses of nivo ( $3 \mathrm{mg} / \mathrm{kg})$; the grade $\geq 3$ AEs rates were $40 \%, 20 \%$, and $50 \%$, respectively, and the pRRs were $80 \%$ (47\% pCR), $77 \%$ (57\% pCR), and 65\% (23\% pCR). Again, RFS was $100 \%$ in those with a pathological response. These data suggest that ipi $(1 \mathrm{mg} / \mathrm{kg})$ plus nivo $(3 \mathrm{mg} / \mathrm{kg})$ is currently the optimal neoadjuvant ICI regimen.

Neoadjuvant ICI therapy has several potential advantages, beyond seemingly greater efficacy and tumour shrinkage that can improve surgical outcomes. "The neoadjuvant 'window of opportunity' approach is particularly appealing as it enables a thoughtful assessment of response to therapy beyond conventional imaging (assessment of pathological responses) as well as collection of tissue samples for critical biomarker analysis," Wargo concludes.

David Killock

ORIGINAL ARTICLES Blank, C. U. et al. Neoadjuvant versus adjuvant ipilimumab plus nivolumab in macroscopic stage III melanoma. Nat. Med. https://doi.org/10.1038/s41591-018-0198-0 (2018) | Amaria, R. N. et al. Neoadjuvant immune checkpoint blockade in high-risk resectable melanoma. Nat. Med. https://doi.org/10.1038/ s41591-018-0197-1 (2018) |Blank, C. U. et al. OpACIN-neo: a multicenter phase II study to identify the optimal neo-adjuvant combination scheme of ipilimumab (IPI) and nivolumab (NIVO). Ann. Oncol. 29 (Suppl. 8), mdy424.052 (2018) 